\title{
Quantitative Assessment of Neuroprotection against NMDA-Induced Brain Injury
}

\author{
John W. McDonald, ${ }^{*}$ NAncy F. Roeser, $†$ Faye S. Silverstein, $†$ And Michael V. Johnston ${ }^{\prime 1}$ \\ *Neuroscience and Medical Scientist Training Program and $†$ Departments of Pediatrics and Neurology, University of Michigan, \\ Ann Arbor, Michigan 48109; and $\ddagger$ Departments of Neurology and Pediatrics, the Johns Hopkins University School \\ of Medicine and the Kennedy Institute, Baltimore, Maryland 21218
}

In immature rodent brain, unilateral intrastriatal injections of selected excitatory amino acid (EAA) receptor agonists, such as $N$-methyl-D-aspartate (NMDA), produce prominent ipsilateral forebrain lesions. In Postnatal Day (PND) 7 rats that receive a right intrastriatal injection of NMDA (25 nmol) and are sacrificed 5 days later, there is a considerable and consistent reduction in the weight of the injected cerebral hemisphere relative to that of the contralateral side $(-28.5$ $\pm 1.9 \%, n=6$ ). In animals treated with specific NMDA receptor antagonists, the severity of NMDA-induced damage is markedly reduced. We have previously reported that the efficacy of potential neuroprotective drugs in limiting NMDA-induced lesions can be assessed quantitatively by comparison of hemisphere weights after a unilateral NMDA injection. In this study, we compared three quantitative methods to evaluate the severity of NMDA-induced brain injury and the degree of neuroprotection provided by NMDA receptor antagonists. We characterized the severity of brain injury resulting from intrastriatal injections of 1-50 nmol NMDA in PND 7 rats sacrificed on PND 12 by (i) comparison of cerebral hemisphere weights; (ii) assay of the activity of the cholinergic neuronal marker, choline acetyltransferase (ChAT) activity; and (iii) measurement of regional brain cross-sectional areas. The severity of the resulting brain injury as assessed by comparison of hemisphere weights increased linearly with the amount of NMDA injected into the striatum up to $25 \mathrm{nmol}$ NMDA. The magnitude of injury was highly correlated with the degree of reduction in ChAT activity $\left(r^{2}=0.97\right)$. Quantification of neuroprotection against NMDA toxicity by measurement of cerebral hemisphere weight disparities was highly correlated with comparisons of hemisphere and striatal cross-sectional areas $\left(r^{2}=0.98\right)$. The potency and efficacy of the dissociative anesthetic ketamine was easily distinguished from those of two other NMDA recep-

\footnotetext{
${ }^{1}$ To whom correspondence should be addressed at Kennedy Institute, Room 506, 707 North Broadway, Baltimore, MD 21205.
}

tor antagonists (MK-801, $(+)$-5-methyl-10,11-dihydro-5H-dibenzo $[a, d]$ cyclohepten-5,10-immine maleate and CPP, 3-(( \pm )-2-carboxypiperazine-4-yl)-propyl-1phosphonic acid) using this model. These data demonstrate that this model can be used to accurately quantitate NMDA-induced brain injury and evaluate neuroprotective properties of glutamate antagonists in vivo. 1989 Academic Press, Inc.

\section{INTRODUCTION}

Selected EAA receptor agonists are neurotoxic in both the developing $(5,19,28,31)$ and the mature $(6,10,32)$ rat brain. The neurotoxicity of NMDA is markedly enhanced in developing rat brain $(15,19)$. Studies carried out both in vivo and in vitro demonstrate that competitive and noncompetitive antagonists of NMDA receptors protect against neuronal injury produced by NMDA $(4,11,12,20,22,23)$. Antagonism of NMDA receptors similarily limits neuronal injury resulting from hypoxiaischemia $(3,8,12,18,29,33)$.

These observations have raised considerable interest in the potential therapeutic applications of NMDA antagonists. Rapid and sensitive methods to quantitate the in vivo neuroprotective effects of such compounds are needed. Most in vivo models of brain injury have relied on anatomical or biochemical approaches to quantitate the severity of injury and efficacy of neuroprotective agents. Cell counts, histopathologic cross-sectional area measurements, and activity of specific neuronal markers such as ChAT activity and neuron-specific immunoreactivity are commonly used as indices of neuronal injury $(2,6,7,8,18,23)$. Recent in vitro assays of injury using neuronal cell cultures have been refined to provide useful quantitative neuroprotection data $(4,9,16)$. However, results from in vitro studies may not accurately reflect in vivo effects.

We have recently developed an in vivo method for quantification of neuroprotection against NMDA-mediated brain injury in perinatal rats $(20,22)$. This model 
takes advantage of the enhanced toxicity of NMDA in PND 7 rats (19). During this phase of rapid development, focal brain damage results in reduced growth of the injured cerebral hemisphere over a several day survival period. In animals sacrificed 5 days after a unilateral NMDA injection, measurement of disparities between NMDA injected and contralateral cerebral hemisphere weights appears to be a good indicator of brain injury (22).

To determine the validity of hemisphere weight measurements as an index of neuronal injury, we compared measurements of hemisphere weights to two more frequently used methods; measurements of (i) tissue ChAT activity and (ii) regional cross-sectional areas in histopathologic sections. The sensitivity of this model was assessed by comparing the neuroprotective effects of one competitive (CPP) and two noncompetitive (MK-801 ketamine) NMDA receptor antagonists against NMDAmediated brain injury. The results demonstrate that comparison of cerebral hemisphere weights can be used as an accurate method for quantifying the effects of potential neuroprotective compounds.

\section{METHODS}

\section{Experimental Protocols}

PND 7 male and female Sprague-Dawley albino rats, weighing between 12 and $18 \mathrm{~g}$, were used for all experiments. Rats were anesthetized with diethyl ether for 3 min. Intrastriatal injections of NMDA were performed as described below. All animals were sacrificed on PND 12,5 days after intrastriatal NMDA injection.

To determine the relationship between the amount of NMDA injected into the striatum and the degree of resulting brain injury six doses of NMDA were used: 1 nmol, $n=5$; $5 \mathrm{nmol}, n=5 ; 10 \mathrm{nmol}, n=5 ; 17 \mathrm{nmol}, n$ $=5 ; 25 \mathrm{nmol}, n=6$; and $50 \mathrm{nmol}, n=2$. Brain injury was assessed first by comparison of injected and contralateral hemisphere weights as described below and then by assay of ChAT activity in the corpus striatum and dorsal hippocampus bilaterally in the same brains.

To further evaluate the reliability of measurements of hemisphere weights as an index of neuronal injury, hemisphere weight measurements were compared to measurements of regional cross-sectional areas in animals that received NMDA (25 nmol) and one of four doses of MK-801. A single dose of MK-801 (ip injection in $0.05 \mathrm{ml}$ PBS) was administered 30 min prior to intrastriatal injection of NMDA $(25 \mathrm{nmol} / 0.5 \mu \mathrm{l})$. Four doses of MK-801 were used: $0.1,0.25,0.5$, and $1.0 \mathrm{mg} / \mathrm{kg}(n$ $=9,7,13$, and 8 , respectively); NMDA-injected controls received ip injections of an equivalent volume of PBS instead of MK-801 $(n=28)$. Animals were sacrificed 5 days later. Quantitation of neuroprotection by area mea- surements was compared with quantitation using disparities between cerebral hemisphere weights in an additional group of animals (doses of MK-801 tested: 0.1, $0.25,0.5$, and $1.0 \mathrm{mg} / \mathrm{kg} ; n=8,9,6$, and 8 , respectively; controls received intrastriatal NMDA injection and PBS ip, $n=20$ ).

The sensitivity of this model was tested by assessing the neuroprotective effects of ketamine, a noncompetitive NMDA antagonist, against NMDA-mediated brain injury in doses ranging from 12 to $183 \mu \mathrm{mol} / \mathrm{kg}$. Six to ten rats were tested at each of six doses. Also, four to six NMDA-injected, PBS-treated controls were included at each dose tested. Results were compared with previously reported dose-response relationships of neuroprotection against NMDA toxicity for one competitive (CPP) and one noncompetitive (MK-801) NMDA receptor antagonist (22). Neuroprotection was assessed by measurement of cerebral hemisphere weight disparities.

\section{Intracerebral Injections}

Injections were performed as previously described (20, 22). Briefly, PND 7 rats were anesthetized with ether and a midline scalp incision was made to expose the calvarium. Pups were positioned in a plaster of paris mold of the head and body. Intracerebral stereotaxic injections were performed with a 26 -gauge, beveled tip. Hamilton syringe using a Kopf small animal apparatus. Injections were made through a hole pierced in the skull using a 25-gauge needle (the skull is very pliable at this developmental age). The site of injection was determined in preliminary experiments and was chosen to simulate the lesion produced in an established perinatal model of focal hypoxic-ischemic brain injury by unilateral carotid ligation and timed exposure to moderate hypoxia (14, 18). All intracerebral injections were made in the posterior striatum. Using bregma as a landmark, injection site coordinates were AP $2.0 \mathrm{~mm}$ and $\mathrm{L} 2.5 \mathrm{~mm}$, at a depth of $4 \mathrm{~mm}$ from the dura. The needle was left in place for $2 \mathrm{~min}$ following injection to limit leakage.

NMDA was dissolved in $0.01 M$ Tris-buffer, $p H$ 7.4. Pups received varying amounts of NMDA intrastriatally in a volume of $0.5 \mu \mathrm{l}$. Preliminary experiments indicated that $0.5 \mu \mathrm{l}$ is the largest volume that can be accurately and reliably administered intracerebrally in perinatal rats.

The scalp was sutured with $6-0$ nylon suture. Rats were subsequently placed under a warming lamp set to maintain a $37^{\circ} \mathrm{C}$ body temperature for $2 \mathrm{~h}$; maintenance of correct body temperature is critical in this model (data not shown). This period ensured adequate recovery from anesthesia and allowed behavioral observations. Pups were then returned to their mother. Five days later, on PND 12, animals were sacrificed by decapitation. 


\section{Quantification of Brain Injury by Comparison of} Cerebral Hemisphere Weights

The forebrain was separated from the cerebellum and brain stem using a razor blade. The two cerebral hemispheres were separated, weighed, and then either frozen for subsequent biochemical assays or placed in $10 \%$ phosphate-buffered formalin solution for histology.

Hemisphere weight disparities were compared for each animal using the formula $100 *(C-I) / C=\%$ damage, a value that reflects the severity of damage of the injected $(I)$ cerebral hemisphere relative to that of the contralateral $(C)$ hemisphere. Values presented are means \pm SEM.

\section{Choline Acetyltransferase Assay}

Striatal and hippocampal tissues from the same animals used to develop the dose-response relationship for NMDA-mediated brain injury were dissected out on ice and then homogenized 1:20 in $50 \mathrm{~m} M$ Tris $(p H$ 7.4) plus $0.02 \%$ Triton X-100. Brain tissue was assayed for ChAT activity by a modification of a previously described method (27). ChAT activity ( $\mathrm{nmol} / \mathrm{min} / \mathrm{mg}$ protein) was expressed as percentage decrease in the injected hemisphere relative to that of the contralateral hemisphere, $\%$ decrease $=100 *(C-I) / C=\%$ damage.

\section{Cross-sectional Area Measurements}

Whole brains were placed sequentially into $10 \%$ phosphate-buffered formalin (PBF) followed by a $30 \%$ sucrose/PBF solution. Fifty-micrometer frozen coronal brain sections were stained for Nissl substance with cresyl violet. Tissue damage was quantitated by measurement of cross-sectional areas of the corpus striatum and the entire cerebral hemisphere using a video-based computerized image analyzer (Imaging Research, St. Catherines, Ontario). For each animal, measurements were made bilaterally in four coronal sections at the level of the striatum. The four measures for each structure were summed within animals by hemisphere; NMDA injected and contralateral hemisphere values were compared as described above for hemisphere weights. Data were expressed as percentage protection, mean \pm SEM (see below).

\section{Neuroprotection Studies}

NMDA antagonists were administered ip in $0.05 \mathrm{ml}$ phosphate-buffered saline (PBS), $p H$ 7.4. In preliminary experiments, this injection volume was determined to maximize consistency of drug delivery. Pups were treated with neuroprotective compounds $15 \mathrm{~min}$ after intrastriatal injection of $25 \mathrm{nmol}$ NMDA $(20,22)$. This delay was chosen to allow partial recovery from anesthesia and to limit interactions between the ether anesthesia and NMDA antagonists. Animals were sacrificed 5 days later.

Data are expressed as percent protection against NMDA toxicity using the formula:

$$
\begin{array}{r}
\% \text { Protection }=100 *(1-[\% \text { damage drug treated } / \\
\% \text { damage PBS treated }]) .
\end{array}
$$

Percent damage values for experimental groups that received NMDA plus a NMDA receptor antagonist were compared with those of controls that received NMDA and PBS. Percentage protection values were expressed as mean \pm SEM. The SEM was calculated:

SEM $(\%$ protection $)=100 *(\mathrm{SEM}$, drug treated $/$

$$
\% \text { damage PBS treated). }
$$

The doses that produced $50 \%$ protection were determined by probit analysis according to the method of Litchfield and Wilcoxon (17).

\section{RESULTS}

Unilateral intracerebral administration of nanomolar quantities of NMDA in PND 7 rats typically produced tonic and tonic-clonic seizure-like activity, and in animals sacrificed 5 days later, there was unilateral gross hemisphere deformity and confluent brain necrosis in striatum which extended into adjacent areas: neocortex, dorsal hippocampus, and thalamus (Figs. 1A and 1B). Damage in regions adjacent to the striatum probably reflects diffusion of NMDA from the injection site. Tissue damage was usually confined to the injected hemisphere; histologic examination occasionally revealed microscopic injury in the opposite cerebral hemisphere. In animals injected with $25 \mathrm{nmol}$ NMDA, the weight of the injected hemisphere relative to the contralateral hemisphere (percent damage) was reduced by an average of $28.5 \pm 1.9 \%, n=6$ (Fig. 2). Among the animals used in the NMDA dose-response experiments, the mortality rate was equivalent in groups that received $25 \mathrm{nmol}$ NMDA or less ( $13 \%$ mortality, $n=6$ /group). Treatment with NMDA receptor antagonists substantially reduced the mortality rate $(<2 \%$ at intermediate doses/drug). Injection of vehicle produced few signs of brain injury (\% damage $=-0.6 \pm 0.7 \%, n=5$ ).

The severity of brain injury produced by intrastriatal injection of NMDA, percent damage, increased linearly with the amount of NMDA injected up to at least $25 \mathrm{nmol}$ NMDA (Fig. 2). Linear regression analysis indicated an associated correlation coefficient of 0.99 . Doses of NMDA greater than 25 nmol NMDA deviated from this linear relationship. Injection of $50 \mathrm{nmol}$ NMDA occasionally resulted in obvious contralateral hemisphere 

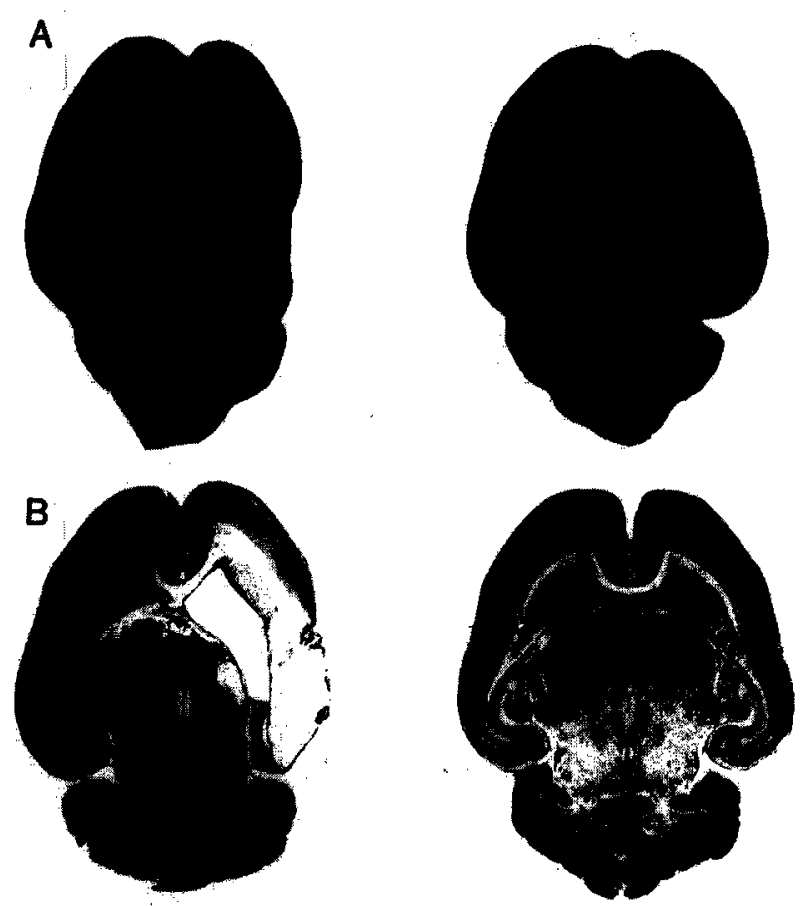

SALINE

MK-801

FIG. 1. NMDA-induced brain injury in brains of 12 -day-old rats that received a unilateral intrastriatal injection of $25 \mathrm{nmol}$ NMDA on PND 7. NMDA was stereotaxically injected into the posterior striatum in a volume of $0.5 \mu$ l. Animals were administered a single dose of either saline or $1 \mathrm{mg} / \mathrm{kg}$ MK-801 intraperitoneally $15 \mathrm{~min}$ after intrastriatal NMDA injection. (A) Representative brains of perinatal rats that received a right intrastriatal injection of NMDA and were treated with either "saline" or "MK-801." Intrastriatal injection of NMDA produced extensive damage in the injected hemisphere (A, saline). There were no obvious signs of injury in the contralateral hemisphere (A, MK-801). (B) Representative Nissl-stained coronal brain sections from the same hrains presented in A. The NMDA-induced lesion is characterized by confluent necrosis in the corpus striatum, extending into the neocortex (B, saline). Damage extends caudally into the dorsal hippocampus and thalamus. In contrast, treatment with $1 \mathrm{mg} / \mathrm{kg}$ MK-801 eliminated obvious signs of neuronal injury (B, MK-801).

injury in addition to the typical ipsilateral damage. With the exception of $50 \mathrm{nmol}$ NMDA, doses of $25 \mathrm{nmol}$ or less did not affect the weight of the noninjected hemisphere relative to that of noninjected controls ( $p=n s$, ANOVA; weights of uninjected hemisphere, control, $415 \pm 15 \mathrm{~g}$ vs NMDA $1 \mathrm{nmol}, 419 \pm 11 ; 5 \mathrm{nmol}, 419 \pm 9 ; 10 \mathrm{nmol}, 413$ $\pm 16 ; 17 \mathrm{nmol}, 384 \pm 19 ; 25 \mathrm{nmol}, 403 \pm 11, n=5-6$ / group; $50 \mathrm{nmol}, 326 \pm 27, n=2$ ). In contrast to the confluent necrosis produced by $25 \mathrm{nmol}$ NMDA (see Fig. 1), injection of 5 or $10 \mathrm{nmol}$ NMDA typically resulted in more selective neuronal necrosis with relative sparing of some neurons.

ChAT activity in the striatum of the same rats used in the NMDA dose-response experiments was reduced by intrastriatal injection of NMDA in a dose-dependent manner (Fig. 3). Similarly, reduction of hippocampal ChAT activity was highly correlated with the amount of NMDA injected $\left(r^{2}=0.88\right)$. However, since the NMDA injection was not delivered directly into the hippocampus, the lowest doses of NMDA did not alter hippocampal ChAT activity. With the exception of $50 \mathrm{nmol}$ NMDA, injection of 1-25 nmol NMDA did not alter ChAT activity in the hemisphere contralateral to the injected hemisphere when compared to that of noninjected controls. The degree of brain injury (expressed as percent damage) resulting from intrastriatal injection of increasing amounts of NMDA was linearly related to the percentage decrease in ChAT activity in both striatum and hippocampus (Figs. 4A and 4B). Linear regression analysis indicated associated correlation coefficients of 0.97 and 0.90 in striatum and hippocampus, respectively.

Intrastriatal injection of $25 \mathrm{nmol}$ NMDA produced a consistent reduction in the cross-sectional areas of the injected striatum and cerebral hemisphere relative to that of the contralateral side. NMDA injected striatal cross-sectional areas were reduced by $59.6 \pm 3.1 \%$ relative to the contralateral striatum, and the cross-sectional areas of the entire cerebral hemisphere at the level of the striatum were reduced by $36.9 \pm 3.2 \%(n=28)$.

We had previously found that the noncompetitive NMDA antagonist MK-801 produced dose-dependent neuroprotection against NMDA-mediated brain injury assessed by comparison of hemisphere weights $(20,22)$. The level of neuroprotection achieved by four doses of MK-801 $(0.1,0.25,0.5,1.0 \mathrm{mg} / \mathrm{kg})$ was similar when quantified by either comparisc $n$ of cerebral hemisphere cross-sectional areas or comparison of cerebral hemisphere weights (Fig. 5). Regression analysis indicated a linear relationship between these two methods of quantifying neuroprotection against NMDA toxicity $\left(r^{2}\right.$ $=0.98$ ). A similar relationship was obtained when crosssectional area values of the striatum were used $\left(r^{2}\right.$ $=0.97$ ).

The dissociative anesthetic ketamine produced dosedependent neuroprotection when increasing dosages were administered ip $15 \mathrm{~min}$ after unilateral intracerebral injection of $25 \mathrm{nmol}$ NMDA (Fig. 6). However, comparison with data reported previously describing the neuroprotective properties of MK-801 and the competitive NMDA antagonist, CPP, indicates that ketamine is a relatively poor neuroprotective agent in this model (22) (Fig. 6). MK-801 was the most potent neuroprotectant with a $50 \%$ protective dose of $0.63 \mu \mathrm{mol} / \mathrm{kg}(95 \%$ confidence limits $=0.22,1.81$ ). Similar values for ketamine and CPP were $113.68(36.30,356.02)$ and 8.84 $(3.93,19.88)$, respectively. The maximal levels of neuroprotection against NMDA toxicity were MK-801, 94.89 $\pm 3.95 \%, 4.17 \mu \mathrm{mol} / \mathrm{kg}$; ketamine, $68.21 \pm 3.4 \%$, 182.66 $\mu \mathrm{mol} / \mathrm{kg}$; and CPP, $84.42 \pm 3.03 \%, 51.38 \mu \mathrm{mol} / \mathrm{kg}$ ). 


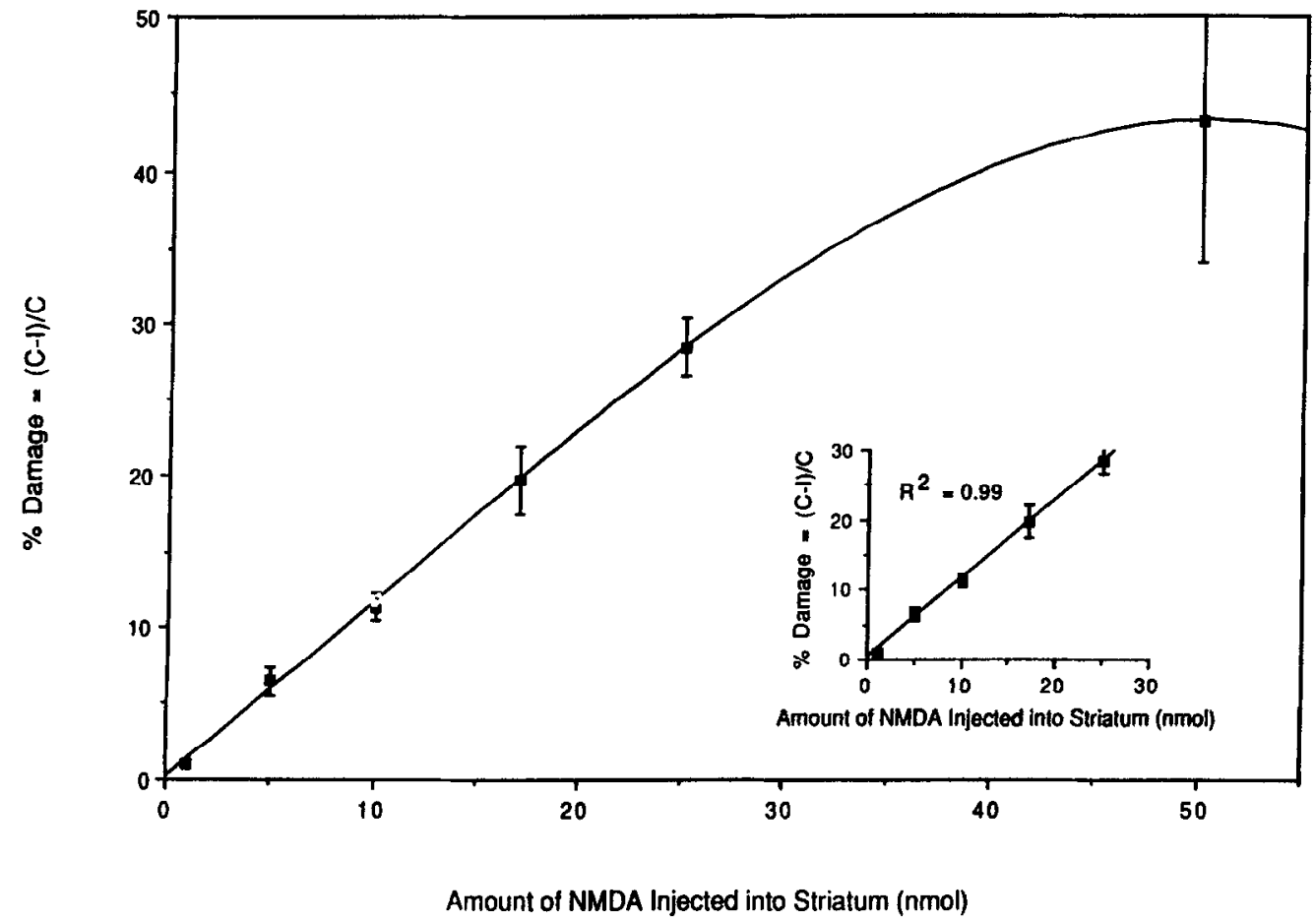

FIG. 2. Relationship between the amount of NMDA injected into the striatum and the degree of resulting brain injury quantitated by measurement of cerebral hemisphere weight disparities. PND 7 rats received unilateral intrastriatal injections of NMDA in amounts increasing from 1 to 50 nmol. Five days later the animals were sacrificed. Brain damage was quantitated by comparison of the weights of the injected (I) and contralateral $(C)$ cerebral hemispheres using the equation indicated on the vertical axis. Brain injury (\%damage) increased linearly with the amount of NMDA injected into the striatum up to at least $25 \mathrm{nmol}$ NMDA. Values represent the means $\pm \mathrm{SEM}: 1 \mathrm{nmol}, n=5 ; 5 \mathrm{nmol}, n$ $=5 ; 10 \mathrm{nmol}, n=5 ; 17 \mathrm{nmol}, n=5 ; 25 \mathrm{nmol}, n=6$; and $50 \mathrm{nmol}, n=2$. The inset illustrates the linear relationship between amount of NMDA injected $(1-25 \mathrm{nmol})$ and $\%$ damage (correlation coefficient $=0.99)$.

Treatment with these neuroprotective compounds reduced the mortality rate to close to zero depending on the dosages used. However, high doses of all these compounds increased mortality above the level of NMDA injected controls. Ketamine dosages greater than $33 \mu \mathrm{mol} /$ $\mathrm{kg}$ resulted in marked mortality rates; $75 \%$ mortality was observed at the maximally protective dose of 183 $\mu \mathrm{mol} / \mathrm{kg}$ and $37.5 \%$ at $120 \mu \mathrm{mol} / \mathrm{kg}$.

\section{DISCUSSION}

Recent studies implicating over activation of EAA receptors in the pathogenesis of neurologic disorders have stimulated the development of drugs designed to limit neuronal injury by blocking activation of these receptors $(13,18,25,26,29)$. Effective and efficient methods to study the neuroprotective properties of these compounds against brain injury in vivo are needed. Quantitation of brain injury is difficult. Established methods used to assess neuronal injury and neuroprotection in vivo have relied on biochemical and anatomical measures of tissue integrity, e.g., measurement of activity of neuronal-specific enzymes, neuronal-specific immunoreactivity, cross-sectional area measurements, and cell counts in lesioned tissue $(2,6-8,19,23)$. We have characterized a perinatal rat model of NMDA-induced brain injury as a method for quantifying the effectiveness of potential neuroprotective compounds by simply comparing the weights of the injected and contralateral cerebral hemisphere weights (22). This study demonstrates that quantitation of brain injury by this method is as sensitive and accurate as two of the more traditional biochemical and anatomical methods cited above. The data presented here indicate that in this model changes in cerebral hemisphere weight closely reflect the extent of neuronal damage.

The severity of brain injury resulting from intrastriatal injection of NMDA, as assessed by hemisphere weight disparities, is linearly related to the amount of NMDA injected into the striatum. In PND 7 rats, injection of $25 \mathrm{nmol}$ NMDA reliably produces about a $30 \%$ decrease in the weight of the injected hemisphere relative to that of the contralateral hemisphere (22). This dose of NMDA maximizes the severity of brain injury while maintaining a low level of mortality ( $13 \%$ mortality rate). The developing brain is more susceptible to NMDA-mediated brain injury than the adult brain (15, 19). NMDA appears to produce about 60 times as much 


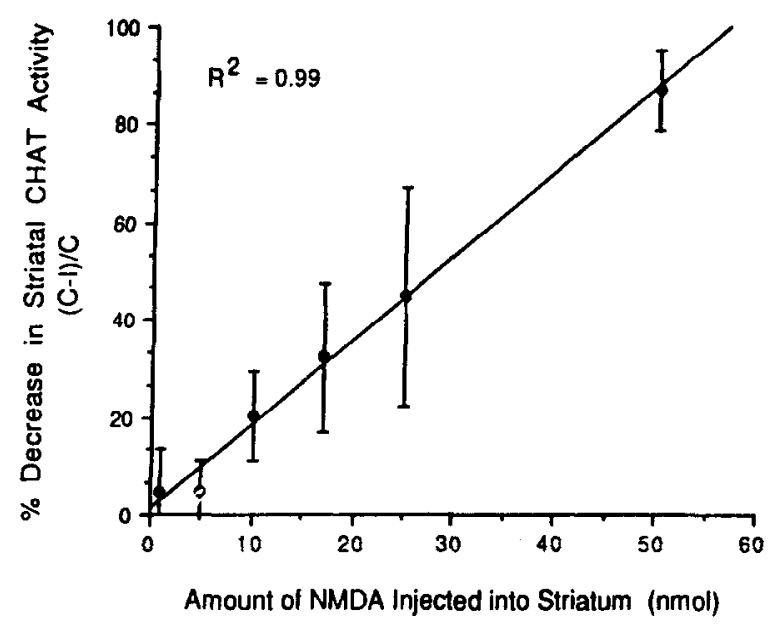

FIG. 3. Relationship between reductions in striatal ChAT activity and amount of NMDA injected intrastriatally in PND 7 rats. Amounts of NMDA from 1 to $50 \mathrm{nmol}$ were injected intrastriatally as described in Fig. 2 and animals were sacrificed 5 days later. ChAT activity was measured bilaterally in the striatum of the same animals included in Fig. 2 (see Materials and Methods). Reductions in ChAT activity in the injected striatum were expressed relative to those in the contralateral striatum using the equation indicated on the vertical axis. Error bars represent the SEM for the number of animals indicated in Fig. 2.

brain damage in 7-day-old rats as compared to adults (19). This enhanced responsiveness to NMDA neurotoxicity facilitates quantification of injury by comparison of hemisphere weights.

The severity of NMDA-mediated brain injury is linearly related to reductions in ChAT activity. Since ChAT activity is a specific marker of cholinergic neu-

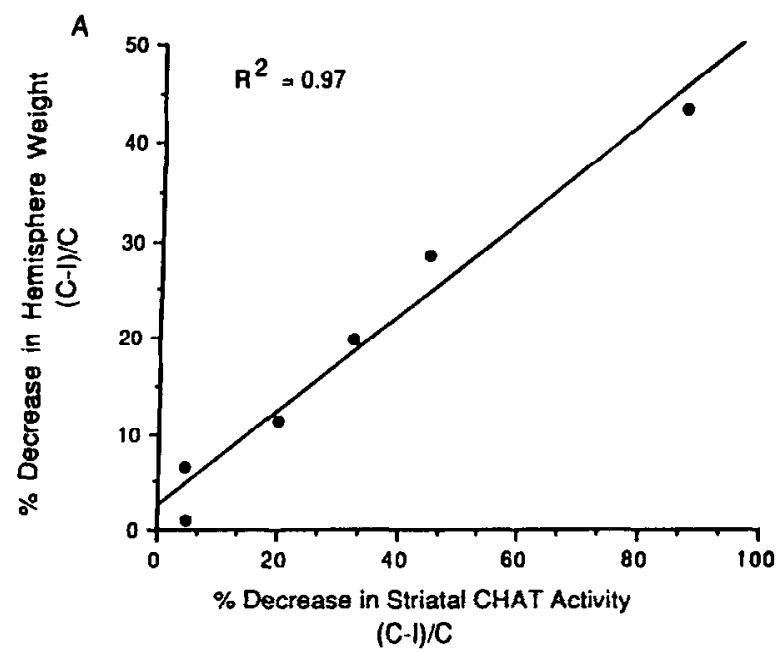

rons, this observation supports the validity of comparison of hemisphere weights of NMDA-injected animals as a quantitative measure of neuronal injury. As well, quantitation of neuroprotection, produced by different doses of MK-801, by comparison of hemisphere weights corresponds closely to similar comparisons of cerebral hemisphere and striatal cross-sectional areas.

This model is a rapid and sensitive method for evaluating the effectiveness of EAA receptor antagonist as well as neuroprotective effects of ions and non-NMDA receptor ligands against NMDA-mediated brain injury (20-22). Potencies and maximal effectiveness of neuroprotective compounds can readily be distinguished. The potencies of NMDA receptor antagonists in this model correspond roughly with their receptor affinities in binding studies as well as with their potencies as anticonvulsants (20-22). However, the potency of ketamine relative to MK-801 is much lower than expected on the basis of binding affinities. This disparity probably reflects effects not present in vitro. Furthermore, it is apparent that the degree of neuroprotection produced by a particular compound is not always related to its anticonvulsant properties (20-22). For instance, MK-801 produces significant levels of neuroprotection at doses that do not block behavioral signs of seizures (22). Also, anticonvulsants such as phenytoin, diazepam, and the calcium antagonist flunarizine do not block NMDA-induced injury in this model even though they prevent seizure activity (20).

Finally, the present model of NMDA-induced brain injury and perinatal rat models of hypoxic-ischemic injury have similar features. The cytopathology of

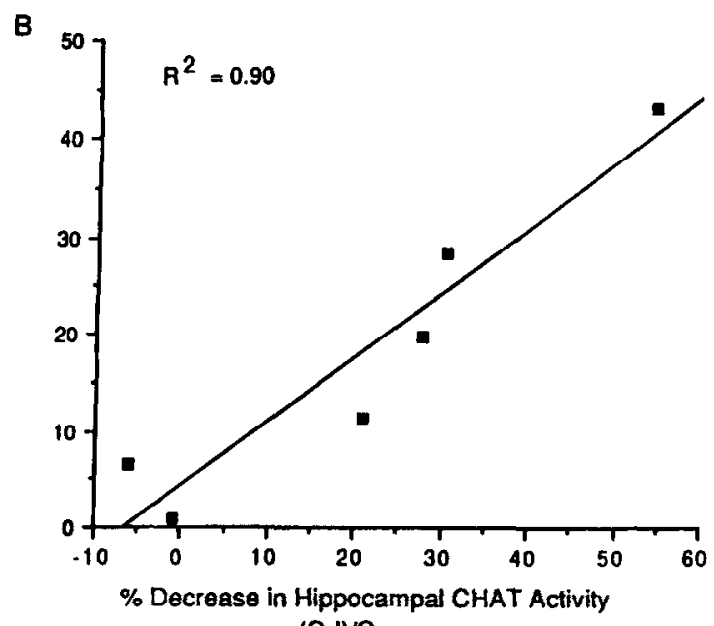

$(\mathrm{C}-\mathrm{I}) / \mathrm{C}$

FIG. 4. Comparison of two methods of quantifying NMDA-mediated brain injury in perinatal rats: measurement of hemisphere weight disparities and ChAT activity. Intrastriatal injections of NMDA (1-50 nmol) were performed as described in Fig. 2. Brain injury was assessed by measurement of cerebral hemisphere weights and by measurement of ChAT activity in the same brains. Data are expressed as percent decrease in the weight or in ChAT activity of the injected hemisphere relative to that of the contralateral hemisphere. Striatal (A) and hippocam pal (B) tissue were used to assay ChAT activity (see Materials and Methods for assay methods). There is a positive linear correlation between percent decrease in cerebral hemisphere weight and percent decrease in ChAT activity. The lines shown were fit by linear regression. 


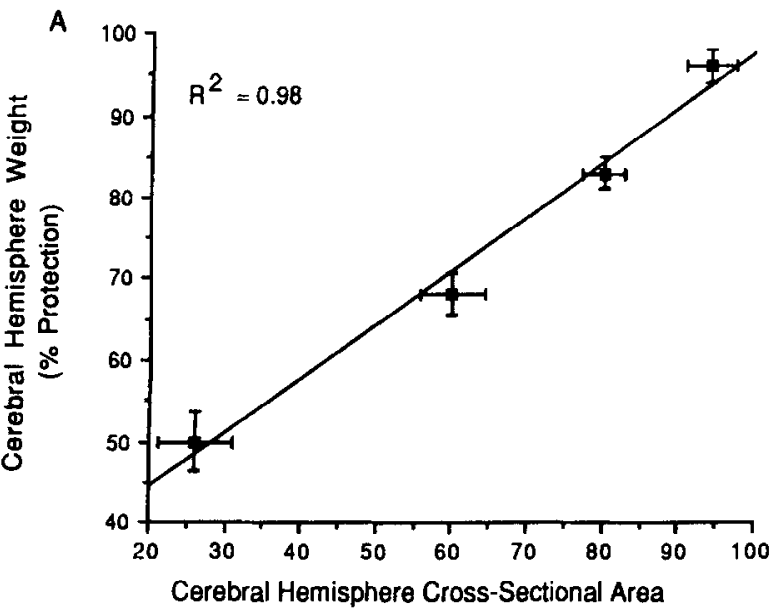

(\% Protection)
B

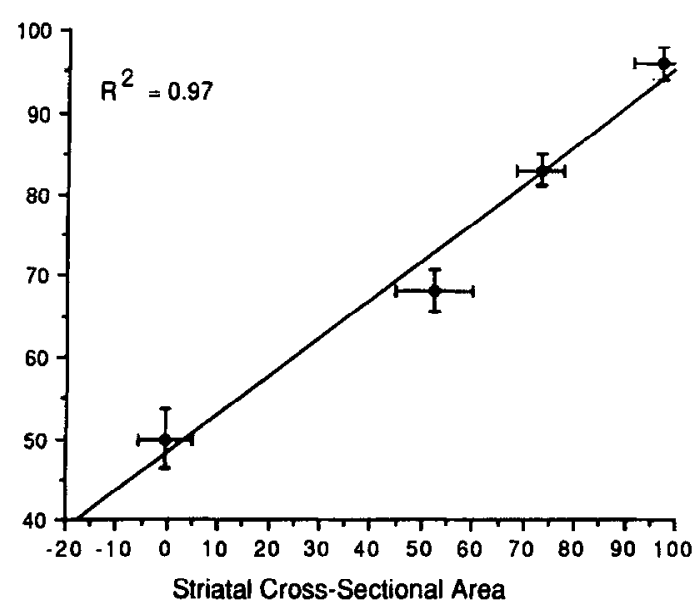

(\% Protection)

FIG. 5. Comparison of two methods for quantification of neuroprotection against NMDA-mediated brain injury. Four doses $(0.1,0.25,0.5$, and $1.0 \mathrm{mg} / \mathrm{kg}$ ) of the noncompetitive NMDA antagonist MK-801 were administered (ip, in $0.05 \mathrm{ml}$ PBS) 30 min prior to unilateral intrastriatal injection of NMDA $(25 \mathrm{nmol} / 0.5 \mu \mathrm{l})$ in PND 7 rats. Five days later animals were sacrificed. The degree of neuroprotection was quantified in independent groups of animals either by comparison of NMDA-injected and contralateral whole hemisphere (A) and striatal (B) cross-sectional areas measured with a video-based image analysis system or by comparison of cerebral hemisphere weights. Linear regression analysis indicated a high degree of concordance between these two methods for quantification of neuroprotection $\left(r^{2}=0.98\right.$, cerebral hemisphere; $r^{2}=0.97$, striatum). Error bars represent SEM. Six to 13 animals per dose were used for each method.

NMDA-mediated brain damage is very similar to that produced by hypoxia-ischemia $(14,18,23)$ and hypobaric-ischemia (15) at this developmental stage. Susceptibility of the brain to injury produced by NMDA tran-

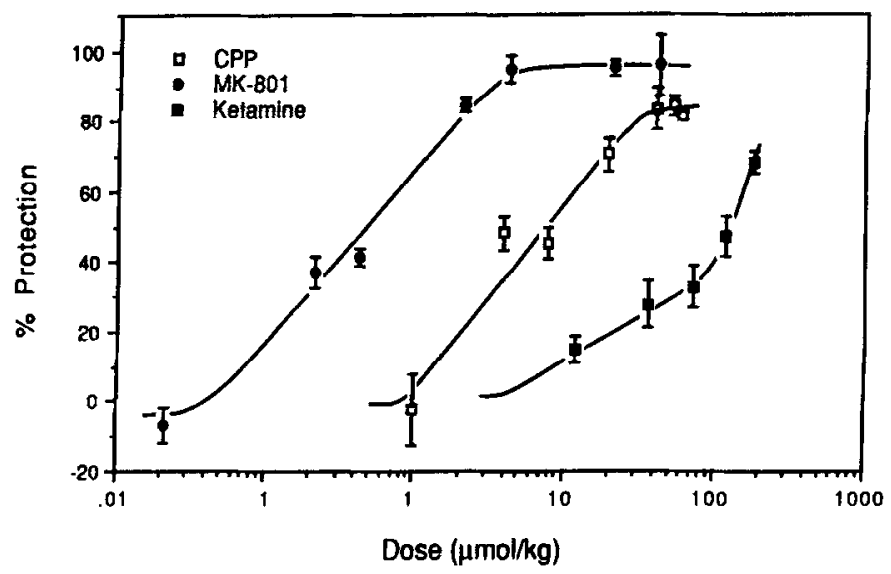

FIG. 6. The quantitative neuroprotective characteristics of ketamine against NMDA-induced brain injury are compared with the effects of noncompetitive (MK-801) and competitive (CPP) NMDA receptor antagonists (22). Brain injury was assessed by comparison of hemisphere weight disparities in the brains of PND 12 rats. Neuroprotective drugs were administered (ip, $0.05 \mathrm{ml} \mathrm{PBS}) 15$ min after intrastriatal injection of NMDA $(25 \mathrm{nmol}, 0.5 \mu \mathrm{l})$ in PND 7 rats $(n=6-10$ per dose). Saline-treated, NMDA-injected littermate controls ( $n=4-$ 6) were included at each dose. Animals were sacrificed 5 days later. Percent protection values were derived by comparison of hemisphere weight disparities of drug-treated and saline-treated groups (see Materials and Methods for details). Values are expressed as means \pm SEM. siently peaks during development around PND 7 in rats (19). Olney and co-workers have recently reported a similar developmental susceptibility to NMDA toxicity and also demonstrated a similar developmental profile for hypobaric-ischemic brain injury $(15,24)$. In general, the effectiveness of drugs in reducing damage induced by NMDA parallels their profile of neuroprotective activity in perinatal hypoxia-ischemia models $(1,20-22,30)$. Thus the activity of compounds in this NMDA model may predict their effectiveness against hypoxic-ischemic neuronal injury.

\section{ACKNOWLEDGMENTS}

This research was supported by USPHS Grants 1PO1NS19613 (M.V.J.) and 1KO8NSO117 (F.S.S.). J.W.M. is a recipient of a MSTP fellowship. We thank S. M. Scholler and H. M. Arft for technical assistance.

\section{REFERENCES}

1. Andine, P., A. Lehmann, K. Ellren, E. Wennberg, I. KJEller, T. Nielsen, AND H. HAGBERG. 1988. The excitatory amino acid antagonist kynurenic acid administered after hypoxic-ischemia in neonatal rats offers neuroprotection. Neurosci. Lett. 90: 208-212.

2. Beal, M. F., N. W. Kowall, K. J. Swartz, R. J. Ferrante, AND J. B. MARTIN. 1988. Systemic approaches to modifying quinolinic acid striatal lesions in rats. J. Neurosci. 8: 3901-3908.

3. Boast, C. A., Gerhardt, S. C., Pastor, G., Lehmann, J., ETiEnNe, P. E., AND Liebman, J. M. (1988). The $N$-methyl-Daspartate antagonists CGS19755 and CPP reduce ischemic brain damage in gerbils. Brain Res. 442: 345-348. 
4. ChOI, D. W., J. Y. KoH, AND S. Peters. 1988. Pharmacology of glutamate neurotoxicity in cortical cell culture: Attenuation by NMDA antagonists. J. Neurosci. 8: 185-196.

5. COOK, T. M., AND K. A. CRU'TCHEK. 1986. Intrahippocampal injection of kainic acid produces significant pyramidal cell loss in neonatal rats. Neuroscience 18: 79-92.

6. CoYLE, J. T., AND R. SCHWARCz. 1976. Lesion of striatal neurones with kainic acid provides a model for Huntingtons chorea. Nature (London) 263: 244-246.

7. DAM, W. M. 1979. The density of neurons in the human hippocampus. Neuropathol. Appl. Neurobiol. 5: 249-264.

8. GiLl, R., A. C. Foster, AND G. N. WoodrufF. 1987. Systemic administration of MK-801 protects against ischemia-induced hippocampal neurodegeneration in the gerbil. J. Neurosci. 7(10): 3343-3349.

9. FinKBeINeR, S., AND C. F. STEVENS. 1988. Application of quantitative measurements for assessing glutamate neurotoxicity. Proc. Natl. Acad. Sci. USA 85: 4071-4074.

10. Foster, A. C., J. F. Collins, and R. Schwarcz. 1983. On the excitotoxic properties of quinolinic acid, 2,3-piperidine dicarboxylic acids and structurally related compounds. Neuropharmacology 22: 1331-1342.

11. Fos'ter, A. C., R. Gill, J. A. KemP, AND G. N. WoOdRUFf. 1987. Systemic administration of MK-801 prevents $N$-methyl-Daspartate induced neuronal degeneration in rat brain. Neurosci. Lett. 76: 307-311.

12. GoldberG, M. P., V. Viseskul, AND D. W. Chol. 1988. Phencyclidine receptor ligands attenuate cortical neuronal injury after $N$-methyl-D-aspartate exposure or hypoxia. J. Pharmacol. Exp. Ther. 245: 1081-1087.

13. Greenamyre, J. T. 1986. The role of glutamate in neurotransmission and neurological disease. Arch. Neurol. 43: 1058-1063.

14. JoHNSTON, M. V. 1983. Neurotransmitter alterations in a model of perinatal hypoxic-ischemic brain injury. Ann. Neurol. 13: 511-517.

15. IKonomidou, C., J. Mosinger, K. Shahid Salles, J. LabruYERF, AND J. W. OLNFY. 1988. Parallel patterns of hypersensitivity to NMA toxicity and hypobaric/ischemic damage in developing rat brain. Soc. Neurosci. Abstr. 14: 501.

16. КоH, J. Y., AND D. W. CHQI. 1987. Quantitative determination of glutamate mediated cortical neuronal injury in cell culture by lactate dehydrogenase efflux assay. J. Neurosci. Methods 20: 8390.

17. LiTCHFIELD, J. T., AND F. WiLCoxoN. 1949. A simplified method of evaluating dose-effect experiments. J. Pharmacol. Exp. Ther. 96: $99-113$.

18. MCDonald, J. W., F. S. Silverstein, ANd M. V. Johnstun. 1987. MK-801 protects the neonatal brain from hypoxic-ischemic damage. Eur. J. Pharmacol. 140: 359-361.

19. McDonald, J. W., F. S. Silverstein, and M. V. Johnston. 1988. Neurotoxicity of $N$-methyl-D-aspartate is markedly en- hanced in the developing rat central nervous system. Brain Res. 459: 200-203.

20. McDonald, J. W., F. S. Silverstein, and M. V. Johnston. 1988. Comparison of neuroprotective effects of competitive and noncompetitive NMDA antagonists against NMDA mediated neurotoxicity in an in vivo perinatal rat model. Pages 601-604 in E. A. Cavalheiro, J. Lehmann, and L. Turski, Eds., Frontiers in Excitatory Amino Acid Research, Vol. 46. A. R. Liss, New York.

21. MCDonald, J. W., F. S. Silverstein, D. CArdona, J. UCKele, R. CHEN, AND M. V. Johnston, 1988. Neuroprotective effects of MK-801 and other compounds on perinatal hypoxic-ischemic injury. Pages 697-707 in E. F. Domino and J-M. Kamenka, Eds., Sigma and PCP like Compounds as Molecular Probes in Biology. NPP Press, Ann Arbor.

22. MCDonald, J. W., F. S. Silverstein, AND M. V. Johnston. 1989. Neuroprotective effects of MK-801, TCP, PCP and CPP against $N$-methyl-D-aspartate induced neurotoxicity in an in vivo perinatal rat model. Brain Res. 490: 33-40.

23. McDonald, J. W., F. S. Silverstein, D. Cardona, C. Hudson, R. CHEN, AND M. V. JoHNSTON. 1989. Systemic administration of MK-801 protects against $N$-methyl-D-aspartate and quisqualate mediated neurotoxicity in perinatal rals. Neuroscience, in press.

24. Olney, J. W., C. IKonomidou, J. Mosinger, K. Shahid Salles, AND G. FRIERDICH. 1988. Role of the $N$-methyl aspartate receptor in developmental psychoneuropharmacology. Soc. Neurosci. Abstr. 14: 417.

25. Rothman, S. M., AND J. W. OLNEy. 1986. Glutamate and the pathophysiology of hypoxic-ischemic brain damage. Ann. Neurol. 19: 105-111.

26. RothMAN, S. M., AND J. W. OLNEY. 1987. Excitotoxicity and the NMDA receptor. Trends Neurosci. 10(7): 299-301.

27. SCHRIER, B. K., AND L. SHUSTER. 1967. A simplified radiochemical assay for choline acetyltransferase. $J$. Neurochem. 14: 977985.

28. Silverstein, F. S., R. C. Chen, AND M. V. Johnston. 1986. The glutamate agonist quisqualic acid is neurotoxic in striatum and hippocampus of immature rat brain. Neurosci. Lett. 71:13-18.

29. Simon, R. P., J. H. Swan, T. Griffiths, and B. S. Meldrum. 1984. Blockade of $N$-methyl-D-aspartate receptors may protect against ischemic damage in the brain. Science 226: 850-852.

30. Simon, R. P., R. S. Young, S. Stout, AND J. CHENG. 1986. Inhibition of excitatory neurotransmission with kynurenate reduces brain injury in neonatal anoxia. Neurosci. Lett. 71: 361-364.

31. Steiner, H. X., G. J. MCBean, C. KoHLER, P. J. Roberts, AND R. SCHWARCZ. 1984. Ibotenate-induced neuronal degeneration in immature rat brain. Brain Res. 307: 117-124.

32. Stewart, G. R., M. Price, J. Olney, B. K. Hartman, and C. CozZARI. 1986. $N$-Methylaspartate: An effective tool for lesioning basal forebrain cholinergic neurons of the rat. Brain Res. 369: 377-382.

33. Weiss, J., M. P. Goldberg, AND D. W. Chol. 1986. Ketamine protects cultured neocortical neurons from hypoxic injury. Brain Res. 380: 186-190. 